\title{
ULTIMATE SENSITIVITY AND PRACTICAL PERFORMANCE OF THE TELLURIUM PHOTOCONDUCTIVE DETECTOR*
}

\author{
D. F. Edwards and M. Mercado $\dagger$ \\ The University of Michigan, Willow Run Laboratories, \\ Ann Arbor, Michigan, U.S.A.
}

\begin{abstract}
Calculations of the ultimate sensitivity and measurements of the practical performance have been made for the tellurium photoconductive detector. For the condition that detector sensitivity is limited by fluctuations of background radiation, the theoretical NEP (noise equivalent power) at the peak of spectral sensitivity $(\lambda=3.4 \mu)$ was calculated to be $5.1 \times 10^{-13} \mathrm{~W}$. For the "best" tellurium detector the measured value of NEP was $3.1 \times 10^{-13}$ $\mathrm{W}$ at the same wavelength and at the optimum chopping frequency. These values indicate that the tellurium photoconductive detector is background limited and therefore is ideal.
\end{abstract}

\section{INTRODUCTION}

THE purpose of this paper is to report the results of calculations of the ultimate sensitivity of the tellurium detector and of measurements of sensitivity for a select few of these detectors as prepared by Suits et al. The results indicate that the tellurium detector is an ideal detector. The fact that only one of six detectors measured approached the ultimate sensitivity indicates the need for continued development. Of the other five, two detectors had an NEP (noise equivalent power) at $3.4 \mu$ within a factor of about ten of the ideal. For the ideal detector, i.e., one that is background-radiation limited, the addition of a cooled radiation shield to match the aperture of the detector to that of the optics should increase the detector sensitivity by as much as a factor of ten.

The use of tellurium as a photoconductor was first investigated in 1949 by Moss, ${ }^{(1,2)}$ who used evaporated thin films. The most sensitive cell that he reported, when cooled to liquid-nitrogen temperature, had a signal equal to the r.m.s. (root mean square) noise in $1 \mathrm{c} / \mathrm{s}$ bandwidth for incident radiation of $1.2 \times 10^{-10} \mathrm{~W}$ of monochromatic radiation at $1 \mu$. This quantity is called the NEP and is defined as the incident radiation necessary to produce a signal equal to the r.m.s. noise. This same tellurium film was only moderately sensitive (NEP $=10^{-6}$ to $10^{-7} \mathrm{~W}$, at $1 \mu$ and $1 \mathrm{c} / \mathrm{s}$ bandwidth) at room temperature. Loferski ${ }^{(3)}$ in 1954 made photoconduclivity measurements on single crystals of tellurium with about the same results as those obtained for evaporated films. Loferski's data were extrapolated and the NEP was found to be about $10^{-8}$ to $10^{-9} \mathrm{~W}$ for the most sensitive photoconductor when cooled to liquid-oxygen temperature. The single-crystals used for Loferski were cut or cleaved from large tellurium crystals, ground, polished, and sometimes etched to the desired dimensions.

Suits ${ }^{(4)}$ in 1957 found that thin hexagonal single-crystal prisms of tellurium when cooled to liquid-nitrogen temperature had sensitivities and response times comparable to those of the lcad salt detectors. These thin single-crystal prisms were grown from the vapor phase in

* This work was conducted by Project MICHIGAN under Department of the Army Contract (DA-36039-78801) administered by the U.S. Army Signal Corps.

$\dagger$ Presently employed at Bendix Aviation Corporation Systems Division, Ann Arbor, Michigan. 
a low-pressure hydrogen atmosphere, starting with $99.999+$ per cent pure material*. The electrical contacts were made by welding a hot wire, in most cases platinum, to the tellurium. The crystals were then mounted in a more or less standard glass dewar with a sapphire window.

\section{ULTIMATE SENSITIVITY}

The ultimate sensitivity was calculated for the tellurium detector for the condition that fluctuation in the background radiation is the fundamental limitation of detector sensitivity. Recently, several articles have been published ${ }^{(5-8)}$ that describe methods for calculating the ultimate sensitivity of a photoconductor for the background-limited condition. The calculations reported in the present paper follow closely those of Moss. ${ }^{(6)}$

The background radiation is taken as a $300{ }^{\circ} \mathrm{K}$ blackbody with the spectral distribution given in Fig. 1, curve (a). Only that radiation that lies within the sensitive spectral region of

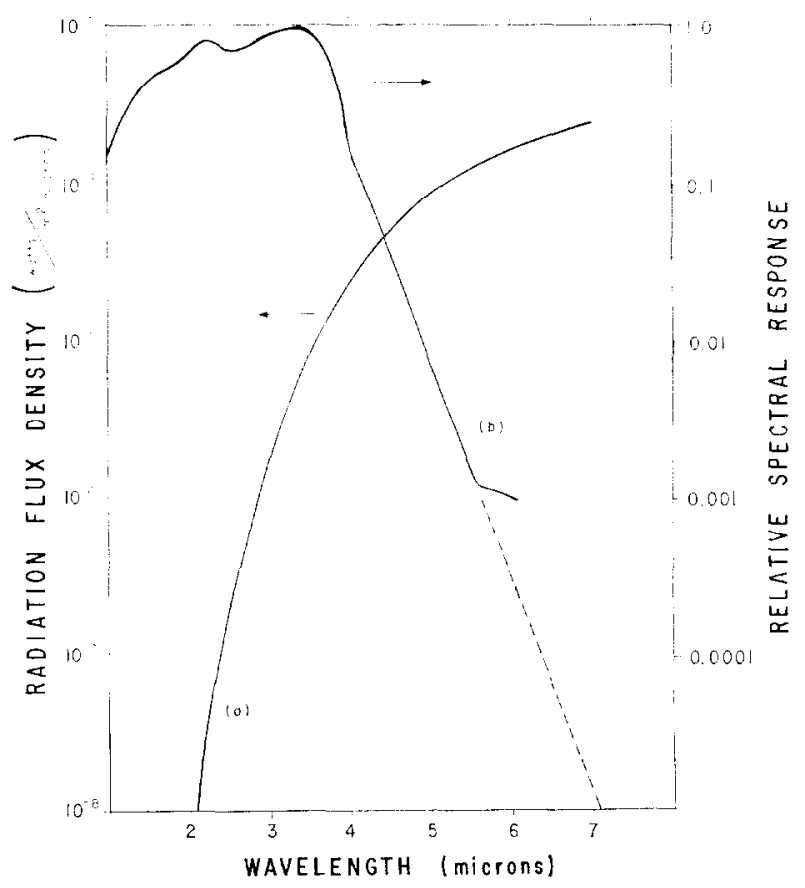

FIG. 1. Curve (a) Spectral distribution for a $300^{\circ} \mathrm{K}$ blackbody. Curve (b) Relative spectral response of the tellurium detector.

the detector will affect the ultimate sensitivity. The relative spectral response of the tellurium detector at $77^{\circ} \mathrm{K}$ is given in Fig. 1, curve (b). The product of these two curves is the spectral distribution of the effective radiation incident on the detector (Fig. 2). It is seen that the effective wavelengths are limited to a narrow band centered at $3 \cdot 7 \mu$. For purposes of calculations, it is assumed to a good degree of approximation that the effective radiation is monochromatic with the wavelength of the peak $3 \cdot 7 \mu$. The area under this

* American Smelting and Refining Co., South Plaintield, New Jersey. 


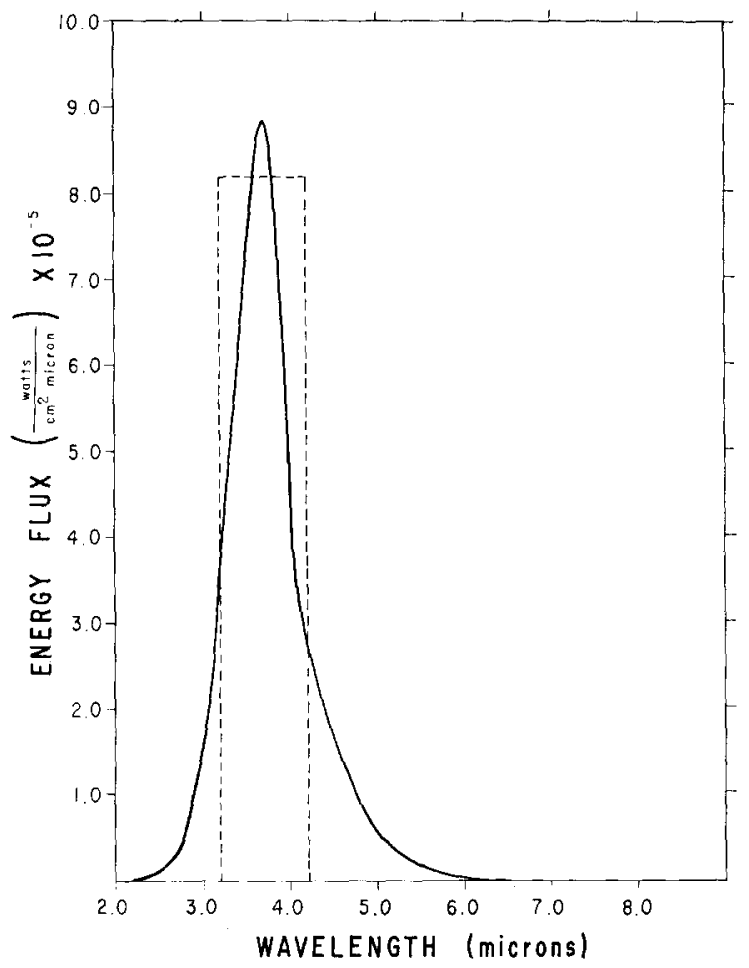

FIG. 2. Spectral distribution of effective radiation incident on detector.

curve is the equivalent energy falling on the detector and equals $82 \cdot 2 \mu \mathrm{W} / \mathrm{cm}^{2}$. This corresponds to $N_{0}=1.5 \times 10^{15}$ quanta/ $/ \mathrm{cm}^{2} / \mathrm{sec}$ at $3.7 \mu$. The number of absorbed quanta producing a detector signal, $N_{s}$, will depend on the absorption properties of the material, the quantum efficiency, and the diffusion length of the free carriers. The relation between $N_{s}$ and $N_{0}$ has been given by Moss ${ }^{(6)}$ as

$$
N_{s}^{2}=2 A N_{0} \Delta f(1-r-t),
$$

where $A$ is the sensitive area of the detector, $\Delta f$ the bandwidth of the measuring equipment, and $r$ and $t$ are the reflexion and transmission coefficients. For a detector with $A=0.0025$ $\mathrm{cm}^{2}$ and $\Delta f=5 \mathrm{c} / \mathrm{s}, N_{s}=9.55 \times 10^{6}$ quanta $/ \mathrm{sec}$ at $3.7 \mu$. The reflexion coefficient $r(=0.48)$ was calculated from the weighted average for the index of refraction, ${ }^{(9)} \vec{n}=5 \cdot 5$. The transmission coefficient $t(=0 \cdot 10)$ was calculated from the absorption coefficient value of Loferski $^{(3)}$ and the sample thickness. This calculated value of $N_{s}$ corresponds to a theoretical $\operatorname{NEP}(3.4 \mu)=5.1 \times 10^{-13} \mathrm{~W}$ at the peak of the spectral response $(\lambda=3.4 \mu)$ and represents the ultimate sensitivity of the tellurium detector for the background-radiation limited condition.

\section{PRACTICAL PERFORMANCE}

The absolute spectral sensitivity of the "best" tellurium photoconductor measured is shown in Fig. 3. The measured NEP at $3.4 \mu$ is $(3 \cdot 1 \pm 0.8) \times 10^{-13} \mathrm{~W}$. This was measured 


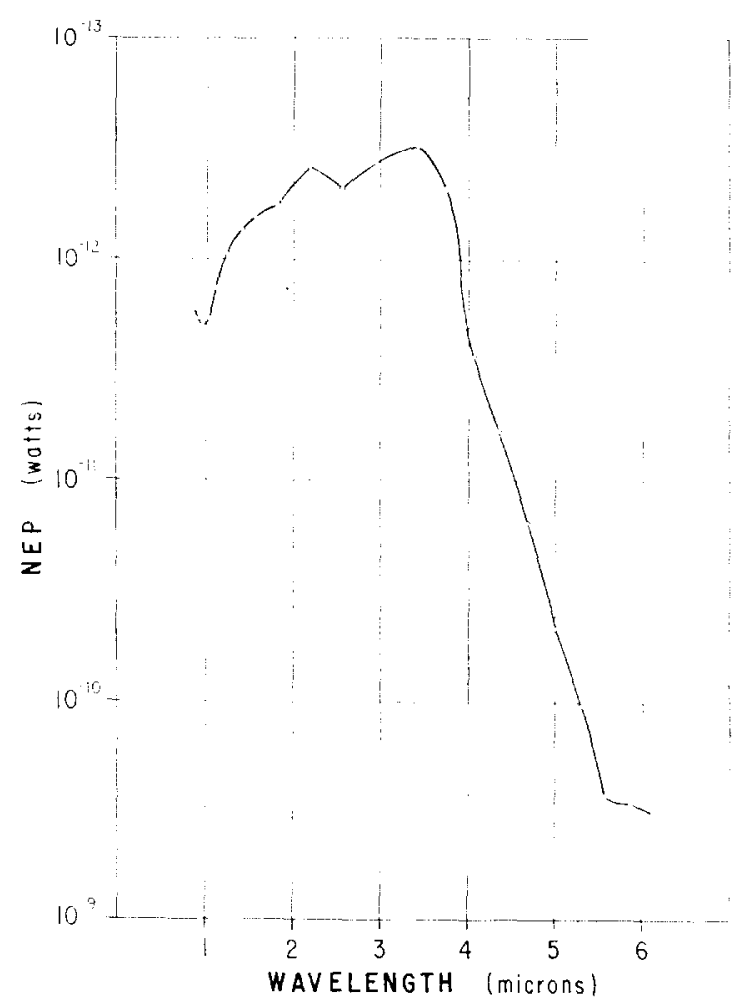

Fir. 3. Absolute spectral sensitivity of the tellurium detector at $77^{\circ} \mathrm{K}$ with a chopping frequency of $5000 \mathrm{c} / \mathrm{s}$ and $5 \mathrm{c} / \mathrm{s}$ bandwidth.

for radiation chopped at $5000 \mathrm{c} / \mathrm{s}$ and $5 \mathrm{c} / \mathrm{s}$ bandwidth. The assumptions used in the calculation of the ultimate sensitivity result in the value of the ideal NEP being larger than the measured value. A more refined calculation does not seem warranted at this time. The monochromatic radiation calibration was made with a Reeder* thermocouple that had been calibrated with a $500{ }^{\circ} \mathrm{K}$ standard blackbody $\uparrow$ and checked with a National Bureau of Standards calibrated lamp. The r.m.s. values of noise and signal voltages were calibrated with a Weston a.c. thermocouple.

\section{REFERENCES}

1. Moss, T. S., Proc. Phys. Soc., Lond. A 62, 264 (1949).

2. Moss, T. S., Photoconductivity in the Elements, Butterworth, London (1952).

3. Loferski, J. J., Phys. Rev. 93, 707 (1954).

4. Suits, G. H., "Tellurium Infrared Detector," University of Michigan, Internal Report (1957).

5. Burstein, E., and G. S. Picus, "Background Limited Infrared Detection," Naval Research Laboratory Internal Report (1958).

6. Moss, T. S., J. Opt. Soc. Amer. 40, 603 (1950).

7. Petritz, R. L., Photoconductivity Conference, Atlantic City. John Wiley, New York (1956).

8. Smith, R. A., F. E. Jones, and R. P. Chasmar, The Detection and Measurement of Infrared Radiution. Oxford University Press, London (1957).

9. Hartig, P. A., and J. J. Loferski, J. Opt. Soc. Amer. 44, 17 (1954).

* Charles M. Reeder Co., Detroit 3, Michigan.

$\uparrow$ Models RS-1A Blackbody, Barnes Engineering Co., Stamford, Connecticut. 